\title{
Communication Challenges in Providing Self-management Education Support to Patients with Type 2 Diabetes
}

\author{
Dr. Ali Alhaiti ${ }^{1} \quad$ Dr. George Lenon ${ }^{2} \quad$ Dr. Mohammad Alrashidi ${ }^{3} \quad$ Dr. Mishal Aldaihani ${ }^{4}$ \\ 1.Education Consultant King Fahad Medical City, Saudi \\ 2.lecturer at RMIT University, Australia \\ 3.Trainer at public authority of apply training and education, Kuwait \\ 4.Director of scholarship and training and education, Saudi
}

\begin{abstract}
This exploratory qualitative study was conducted to examine the perceptions of nurses, health educators, and dieticians regarding care for patients with type 2 diabetes mellitus. The findings of this study highlighted language barriers and patient engagement as the most common communication challenges.
\end{abstract}

Keywords: Type 2 diabetes, Care management, Communication, Saudi Arabia

DOI: $10.7176 / \mathrm{JHMN} / 87-05$

Publication date:March $31^{\text {st }} 2021$

\section{Introduction}

Maintaining patient interaction to deliver highly skilled care is an essential aspect of nursing practice in any clinical setting. Therefore, enhancing communication and engagement skills is crucial for nursing staff to establish productive interactions with patients. According to Pung and Goh (2017), one of the limitations of communication in a multicultural environment is the language barrier that manifests during direct nursing interaction with patients and engagement work in the care process.

Failure to establish productive communication due to language barriers affects the quality of care. Almualem, Darwish, and AlFaraj (2021) described anxiety as one of the manifestations of poor interaction since patients cannot receive adequate advice or support if a healthcare professional cannot verbally explain certain nuances of treatment or care. Communication is the most important aspect in determining nursing qualifications (Ali \& Watson, 2018). Arabic is the official language of Saudi Arabia, an advanced country with modern healthcare and labour-intensive approaches. Thus, foreign specialists who do not have sufficient language training will experience challenges that manifest during patient-provider interactions. As Alshammari, Duff, and Guilhermino (2019) argue, "the barriers to effective nurse-patient communication adversely effects patient safety and patient satisfaction" (p. 1). Therefore, addressing this limitation is of great importance in order to promote a high level of healthcare services.

The personal views of participants involved will be used as a rationale for collecting data on language barriers and engagement challenges. Establishing interactions with the target population is a prerequisite for patient adherence to care guidelines (Mani \& Abutaleb, 2017). Moreover, due to the lack of productive interaction caused by communication gaps, some patients feel distrust of the professionalism of the staff, which manifests itself as an unwillingness to perform the prescribed procedures responsibly and timely. Bit-Lian, Bakar, and Saeidin (2020) described Saudi culture and its commitment to traditions and indigenous behaviours. As a result, the foreign background of nurses involved as participants was a deterrent to productive communication. The scope of this study includes assessments of communication challenges between nursing staff and patients with type 2 diabetes (T2D), in particular, ensuring effective self-management education support.

\section{Method}

This study included face-to-face semi-structured interviews with nurses and clinical educators from the King Fahad Medical City (KFMC) and Specialised Diabetes and Endocrine Centre (SDEC). Using open-ended questions allowed the participants to express their perceptions using their own words and provided opportunities for the participants to introduce aspects of the topic that might not be discussed if the researcher had opted to use close-ended questions (Irvine, Drew \& Sainsbury, 2013). The use of open-ended questions was also recommended to allow participants to respond to the questions with as much or as little detail as they felt comfortable with (Cachia \& Millward, 2011). Aside from this, the use of a semi-structured interview guide helped the researcher ensure that all aspects of the topic relevant to the study were discussed during the interview and all information needed to address the research questions was collected (Doody \& Noonan, 2013).

\section{Recruitment}

The researcher recruited participants by inviting nurses and clinical educators who indicate their willingness to participate in this study were asked to tick the option for interview and provide contact details to the researcher. The researcher contacted the nurses and clinical educators using the details provided to schedule an appointment 
for an individual interview. The initial plan was to recruit 10 interview participants, but this was increased to 13 participants to reach data saturation. The interviews were held at a private office in the hospital, which was reserved by the researcher for this purpose.

On the day of the scheduled interview, the researcher opened the session by reviewing the policies and procedures identified in the informed consent form. The researcher reiterated that the interviews were audio recorded. After reviewing the informed consent form, the researcher asked the asked who wish to participate in the interview to sign the form, including a waiver for the recording of the interview and the subsequent transcript. Once the informed consent form was signed, the researcher began the interview. After each interview, the researcher transcribed the recording and sent a copy of the transcript to the participants for their review and approval. This step was undertaken to enhance the validity and reliability of the study results (Ali \& Yusof, 2011). Once all the transcripts had been approved, the researcher began the qualitative data analysis procedures.

\section{Inclusion Criteria}

To be eligible for this study, nurses had to meet the following inclusion criteria: (1) 21-60 years of age, (2) a nurse at the KFMC and SDEC, (3) working at the SDEC Diabetes Centre for at least one year and (4) able to read and write in Arabic or English.

\section{Exclusion Criteria}

Based on the inclusion criteria described above for this study, nurses who met the following descriptions were excluded from participating in this study: (1) less than 20 or more than 60 years of age, (2) employed in KFMC, but not in the SDEC Diabetes Centre, (3) worked for less than one year in the SDEC Diabetes Centre and (4) cannot read and write in Arabic or English.

\section{Data Analysis}

The qualitative data was analysed using content analysis. It is one of the most widely used analytical tools due to its flexibility in identifying codes deductively (top-down, theory driven) or inductively (bottom-up, data driven), depending on the topic and the nature of the interviews (Leech \& Onwuegbuzie, 2011).

The specific procedures used were in accordance with Thomas's (2006) guidelines. First, the raw data were prepared in text form and all transcripts were formatted consistently (data cleaning). Then, each transcript was read several times to ensure familiarity with the data and highlight recurring codes and emerging themes in the text. Both general (or meta) categories (based on the research questions), and more specific categories were created (in vivo coding, based on the raw data). These specific categories differed as little as possible from the participants' own words; given the large amount of data, NVivo was used here to aid in this analytical process. Next, overlapping codes were assessed to ensure that they could be categorised under similar themes, and uncoded texts were evaluated to ensure that they were not relevant to the research questions.

\section{Result}

\section{Overview of the Participants}

Thirteen members of a multidisciplinary team operating the Diabetes Centre took part in this interview. Eight of the participants were nurses, four worked as health educators and one worked as a dietician. Five of the participants were from Saudi Arabia, two were from India and six were from the Philippines. The majority of participants had more than 11 years of employment experience, while three had less than 2 years of experience. With regard to experience working specifically with type 2 diabetes (T2D) patients, none of the participants had more than 12 years experiences; nine had less than 2 years; and four had 2-6 years of experience in this field.

All the participants in this study held a bachelor's degree in their discipline (nursing, health education or clinical dietician) obtained from a local university in their country of origin. None of the participants had any postgraduate training. The degree of professional training varied between participants, depending on their availability and internal management criteria for each employee. Those who specialised in nursing had much more experience in their field than those from other disciplines; they also completed several training courses on supporting patients with T2D.

\section{Participants Response.}

In the interview, participants were asked to discuss the communication challenges they face while providing selfmanagement education support to patients with T2D, a large portion of the interviews was dedicated to this topic, and participants were encouraged to discuss their perceptions in depth. Due to the richness of their responses, their narratives (and associated subthemes). These are summarised in Table 1. 
Table 1

\begin{tabular}{|l|l|l|}
\hline \multirow{2}{*}{$\begin{array}{l}\text { Communication } \\
\text { challenges }\end{array}$} & Language & Not speaking Arabic is a language barrier \\
\cline { 2 - 3 } & Engagement & $\begin{array}{l}\text { Not compliant with treatment } \\
\text { Patients are not committed to following advice } \\
\text { Patients do not believe in our role as professionals } \\
\text { Not compliant with medication, monitoring blood sugar exercise and diet } \\
\text { control }\end{array}$ \\
\hline
\end{tabular}

\section{language}

Many participants felt that the Arabic language is one of the challenges that nurses face, as many nurses are from foreign countries where the native language is not Arabic. Eight of the participants, as mentioned earlier, are nurses and non-native Arabic speakers. Given that language is the primary tool by which knowledge is relayed, nurses are often frustrated by the language barrier, even if they have taken multiple Arabic language courses:

The main problem for me is the language because I cannot speak to them straight [fluent] Arabic. Other patients can understand me when I am speaking Arabic but I need to [communicate in] sign language or sometimes I have to ask my colleagues to explain it to them (Elizabeth, RN).

\section{Engagement}

A number of the participants expressed disappointment with patients' lack of commitment and cooperation. Often, patients do not follow the advice given to them by nurses, clinical educators and dieticians; further, they are often unwilling to take full personal responsibility for their health. According to one of the nurses, some patients are acutely aware of the negative health consequences they might face if they do not follow their advice, but still they do not want to cooperate. Instead, they want to be given medication: 'most patients are already aware of the complications, treatment, and when and how they seek medical advice ... But they are not compliant with these things (exercise, diet and treatment). The main problem is that patients only want their medicines' (Ashley, RN).

Some participants also commented on the lack of awareness about the role of health educators providing self-management education support for diabetes; this included patients' lack of willingness to listen to their educators' full session, their overall reluctance to fully discuss their lifestyle habits, and, in some cases, their complete disregard for the role of HCPs and educators who were not their primary physicians. There was a common perception that patients are not interested in undergoing education interventions, and simply want to meet with the physician who prescribes their medication, after which they hurry out of the clinic:

Some patients come here just to see the doctor and pick up their medication, not to see us ... We keep reminding them to come here, especially the 29 - or 30 -year-old males who are always in a hurry ... Sometimes they say, 'I'm not going to listen to you' or 'please be quick, I'm busy'... In these types of situations, I consider it to be like a competition with myself, so I keep trying many times till they start to believe in me and my role as a dietician (Maya, CE).

The lack of understanding about the importance of the educator's role was described as a major obstacle that clinical educators need to overcome when interacting with patients at the clinic. Often, the patients' reasons for not wanting to undergo an educational intervention is that they either do not have sufficient time, or that they feel they already have adequate knowledge about diabetes and have no desire to learn more about their medical condition.

\section{Discussion}

Saudi cultural and socioeconomic nuances contribute significantly to communication challenges that hinder the effectiveness of self-management education support for controlling T2D. The respondents in this study reported that most nurses working in the diabetes centre were foreigners. According to Al-Khathami Kojan Aljumah et al. (2010), T2D is a global disease that affects people from various parts of the world, both literate and illiterate, which means that when healthcare professionals and patients do not speak the same language, language barriers may hinder the effective delivery of relevant information, skills and knowledge to patients (Karliner, Kim, Meltzer \& Auerbach, 2010). Language barriers contribute to ineffective forms of communication, which limits the understanding of self-management education support for controlling T2D (Elkharam et al., 2013).

Al-Khathami et al. (2010) pointed out that healthcare in Saudi Arabia, particularly nursing care, is largely delivered by a foreign labour force. This is consistent with our findings. The vast majority of nursing professionals in the current study were non-Arabic-speaking individuals from diverse cultural backgrounds. This has contributed to linguistic diversity and a marked language barrier between Arabic-speaking patients and foreign nurses. Consequently, these nurses are unable to comprehensively understand the fears, doubts, and anxieties felt by patients during nursing care delivery.

The nurse participants pointed out reduced engagement from patients, especially in regards to treatment 
compliance. They believed that most patients did not take instructions from their healthcare providers seriously, which limited the delivery of self-management education support. Delamater (2006) pointed out that regimen adherence problems are common in individuals with diabetes. To improve the outcomes of patients with T2D, it is important for patients to comply with information that they receive from their healthcare practitioner (Meuter, Gallois, Segalowitz, Ryder, and Hocking, 2015).

In conclusion, the findings of this study will help to identify the impact of doctor-patient communication on the promotion of lifestyle changes and adherence to medication. According to Ha and Longnecker (2010), it is important for health professionals to effectively communicate with their patients to ensure total adherence to medication and lifestyle changes. However, given that the findings from the present research show that patients do not comply with self-management, it is possible that such communication is ineffective. The other significant aspect of this study is that it can be used to identify self-management practices that should be prioritised with the aim of ensuring patients' adherence. The research also identifies practices that only require reinforcement because the level of adherence is already high.

\section{Recommendation}

The assessment of the existing constraints and barriers can reveal the root causes and prerequisites for patients' reluctance to follow the recommendations of nursing staff. Self-management education support is an aspect to enhance to provide a higher quality of care and create an enabling environment for the well-being of targeted patients. The Arabic language training courses can be ineffective for nurses to gain sufficient knowledge to interact with the community. Therefore, addressing the language barrier is seen as one of the crucial measures to improve the quality of provider-patient communication and create a productive care environment, which is relevant to the treatment of type 2 diabetes as a disease that requires the constant monitoring of health indicators and compliance with medical prescriptions.

\section{Conflict of interest}

The authors declare that they have no conflict of interest.

\section{Acknowledgments}

I would like to express my deep gratitude to my supervisor, Dr George Lenon for his amazing support and playing an important role in this study and for always being optimistic. Extend my thanks and gratitude to my colleague Dr. Mohammad and Dr. Mishal for their contribution in this study.

\section{References}

Al-Khathami, A. M., Kojan, S. W., Aljumah, M. A., Alqahtani, H., \& Alrwaili, H. (2010). The effect of nursepatient language barrier on patients' satisfaction. Saudi Medical Journal, 31(12), 1355-1358.

Al-Khathami, A. M., Kojan, S. W., Aljumah, M. A., Alqahtani, H., \& Alrwaili, H. (2010). The effect of nursepatient language barrier on patients' satisfaction. Saudi Medical Journal, 31(12), 1355-1358.

Ali, A., \& Yusof, H. (2011). Quality in qualitative studies: The case of validity, reliability, and generalizability. Issues in Social and Environmental Accounting, 5(1), 25-64.

Ali, P. A., \& Watson, R. (2018). Language barriers and their impact on provision of care to patients with limited English proficiency: Nurses' perspectives. Journal of Clinical Nursing, 27(5-6), e1152-e1160. https://doi.org/10.1111/jocn.14204

Almualem, J., Darwish, A., \& AlFaraj, A. (2021). The relationship between language barrier in non-Arabic nurses and anxiety in cardiovascular patients: A cross-sectional descriptive study. Journal of Patient Experience, 8, 1-5. https://doi.org/10.1177/2374373521989242

Alshammari, M., Duff, J., \& Guilhermino, M. (2019). Barriers to nurse-patient communication in Saudi Arabia: An integrative review. BMC Nursing, 18(1), 1-10. https://doi.org/10.1186/s12912-019-0385-4

Bit-Lian, Y., Bakar, R. A., \& Saeidin, S. (2020). The perceived cultural barriers to effective communication towards patient among non-Saudi Registered nurses of a public hospital, the Kingdom of Saudi Arabia. The Malaysian Journal of Nursing (MJN), 11(4), 41-53. https://doi.org/10.31674/mjn.2020.v11i04.004

Cachia, M., \& Millward, L. (2011). The telephone medium and semi-structured interviews: A complementary fit. Qualitative Research in Organizations and Management: An International Journal, 6(3), $265-277$. doi:dx.doi.org/10.1108/17465641111188420

Delamater, A. M. (2006). Improving patient adherence. Clinical Diabetes, 24(2), 71-77.

Doody, O., \& Noonan, M. (2013). Preparing and conducting interviews to collect data. Nurse Researcher, 20(5), 28-32. doi:dx.doi.org/10.7748/nr2013.05.20.28.e327

Elkharam, W. M., Khatri, R., Wallymahmed, A. H., Gee, I. L., \& Elhisadi, T. (2013). Knowledge of and adherence to health advice among adults with diabetes in Libya. Ibnosina Journal of Medicine and Biomedical Sciences, 5(3), 140-147. 
Ha, J. F., \& Longnecker, N. (2010). Doctor-patient communication: A review. The Ochsner Journal, 10(1), 3843

Irvine, A., Drew, P., \& Sainsbury, R. (2013). 'Am I not answering your questions properly?' Clarification, adequacy, and responsiveness in semi-structured telephone and face-to-face interviews. Qualitative Research, 13(1), 87-106. doi:10.1177/1468794112439086

Karliner LS, Kim Se, Meltzer dO, auerbach ad. (2010). Influence of language barriers on outcomes of hospital care for general medicine inpatients. J Hosp Med, 276-282.

Leech, N. L., \& Onwuegbuzie, A. J. (2011). Beyond constant comparison qualitative data analysis: Using Nvivo. School Psychology Quarterly, 26(1), 70.

Mani, Z. A., \& Abutaleb, M. (2017). Communication skills of novice nurses at psychiatric hospital in Saudi Arabia. Journal of Nursing and Care, 6(407), 2167-1168. https://doi.org/10.4172/2167-1168.1000407

Meuter, R. F., Gallois, C., Segalowitz, N. S., Ryder, A. G., \& Hocking, J. (2015). Overcoming language barriers in healthcare: A protocol for investigating safe and effective communication when patients or clinicians use a second language. BMC Health Services Research, 15(1), 371.

Pung, L. X., \& Goh, Y. S. (2017). Challenges faced by international nurses when migrating: An integrative literature review. International Nursing Review, 64(1), 146-165. https://doi.org/10.1111/inr.12306

Thomas, D. R. (2006). A general inductive approach for analyzing qualitative evaluation data. American Journal of Evalution, 27(2), 237-246. 